\title{
La Nouvelle Histoire de la PENSEe ÉCONOMIQUe OU LA DIVISION DU TRAVAIL DANS L'HISTOIRE DE
}

\section{LA PENSEE ECONOMIQUE}

Jean-Marc Ponsonnet \& Jean-Nicolas Rieucau ${ }^{1}$

\section{Résumé :}

Outre l'envergure inégalée de son champ d'investigation, la grande qualité de la Nouvelle Histoire de la Pensée Économique réside dans la finesse de la plupart des analyses qui y sont menées, résultat de la collaboration d'une trentaine de spécialistes. Cette écriture à plusieurs mains a cependant sa contrepartie, dans la mesure où, à l'exception des éditeurs, aucun des auteurs ayant participé à cet ouvrage ne l'a manifestement lu dans son intégralité : certaines répétitions, des renvois peu fréquents entre les commentaires et, enfin, une disparité critique prononcée dans le traitement des littératures primaires et secondaires, ne permettent pas au lecteur d'accéder aisément à une approche compréhensive et transversale de l'histoire de la pensée économique ici relatée. Néanmoins, s'il est avisé, il y trouvera une quantité considérable d'informations précises et d'études approfondies.

\section{Abstract : Nouvelle Histoire de la Pensée Économique or the division of labour within the history of economic thought}

Even though its scope remains unprecedented, the main quality of the Nouvelle histoire de la pensée économique lies in the acuteness of most of the studies of which it is composed, which were written by some thirty leading specialists. Such a collective work has its drawbacks, especially since none of the authors - the editors excepted - seem to have read it through integrally. Repetitions are not always avoided, cross-references are scarce, and a marked critical disparity in the treatment of primary and secondary sources does not allow the readers a comprehensive and transversal view of the history of economic thought. Wiser readers however, will find a wealth of precise information and thorough studies.

\section{Classification JEL : B100, B200}

\footnotetext{
${ }^{1}$ Université Paris I, PHARE, jean-marc.ponsonnet@,wanadoo.fr et rieucau@,univ-paris1.fr
} 
« Dans le progrès de la société, la philosophie ou la spéculation devient, comme tout autre emploi, le principal ou le seul métier et occupation d'une classe particulière de citoyens. Comme tout autre emploi aussi, elle est subdivisée en un grand nombre de branches différentes, dont chacune occupe une tribu ou une classe singulière de philosophes; et cette subdivision d'emploi dans la philosophie, comme dans toute autre activité, améliore l'habileté et fait gagner du temps. Chaque individu devient plus compétent dans sa propre branche singulière, il est au total fait plus d'ouvrage, et la quantité de science en est considérablement accrue $»^{2}$.

S'il est de connaissance commune que l'on peut trouver dans l'œuvre d'auteurs aussi divers que Platon, Aristote et plus près de nous, Petty, Mandeville et Ferguson, le germe plus ou moins établi des analyses que Smith mène au sujet de la division du travail, ce dernier semble toutefois faire ouvre novatrice en allant jusqu'à donner un tour systématique à la répartition des tâches dans le domaine de l'activité intellectuelle. Certes, l'exemple de la «Grande » Encyclopédie, dont Smith fut un fervent lecteur et dans laquelle il ira jusqu'à puiser son exemple de la manufacture d'épingles, lui a sans doute fourni matière à réflexion... Quoi qu'il en soit, on ne peut s'empêcher de souligner jusqu'à quel point les historiens de la pensée économique, commentateurs s'il en est de la Richesse des nations, ont tardé à se nourrir eux-mêmes de l'enseignement de Smith. Le principe de la division du travail dont Smith vante les mérites une dizaine d'années après que l'un de ceux qu'il rangeait parmi les «philosophes français $»^{3}$, Dupont de Nemours, a rédigé le premier texte d'histoire de la pensée économique connu à ce jour $^{4}$, a dû effectivement attendre plus de deux cents ans avant de connaître une véritable application dans ce même domaine de l'histoire de la pensée économique.

En ce sens, l'entreprise intellectuelle dirigée par Alain Béraud et Gilbert Faccarello, parce qu'elle réunit les travaux d'une trentaine de chercheurs, constitue réellement une «Nouvelle » histoire de la pensée économique. Unique en son genre par son caractère collectif, ce livre n'a d'autre équivalent, du point de vue de sa densité - plus de mille sept cents pages réparties en trois tomes - et de sa qualité, que la fameuse Histoire de l'analyse économique [1954] de Schumpeter. A cet égard, il est révélateur qu'en tant qu'historien, le nom de Schumpeter constitue une référence privilégiée pour un grand nombre d'auteurs des contributions de la Nouvelle histoire de la pensée économique ${ }^{5}$.

Cependant l'existence de cette épithète "Nouvelle» dérive également d'une précaution méthodologique. Les principaux résultats figurant dans l'ouvrage, est-il précisé dans l'«Avant-propos »

\footnotetext{
${ }^{2}$ Enquête sur la nature et les causes de la richesse des nations [1776, vol. 1, p. 12]. Voir aussi Early Draft of Part of the Wealth of Nations [1763, p. 570] et Lectures on Jurisprudence [1762-1763, p. 347], [1763-1764, p. 492].

${ }^{3}$ Enquête sur la nature et les causes de la richesse des nations [1776, vol. 2, p. 756].

${ }^{4}$ De l'origine et des progrès d'une science nouvelle [1767].

${ }^{5}$ Afin d'illustrer ce point, on exclura les contributions figurant dans le tome III car celui-ci traite dans sa majeure partie de la pensée économique du $\mathrm{XX}^{\mathrm{e}}$ siècle : Schumpeter s'y trouve alors naturellement cité, en général, comme économiste. En revanche, treize des seize études comprises dans les deux premiers tomes font intervenir au moins une fois le nom de Schumpeter considéré comme historien (t. I, p. 15, 16, 37, 43, 176, 204, 228, 410, 512, 518, 524, 531 ; t. II, p. 11, 30, 266, $449,553,598)$. À titre de comparaison, seuls trois commentateurs font référence à l'Histoire des doctrines économiques [1944] de Gide \& Rist (t. I, p. 512, 517, 548 ; t. II, p. 56, 58). Blaug, avec son Histoire de la pensée économique [1985], ne voit quant à lui son nom cité que dans deux contributions (t. I, p. 511, 531, 533, 547). Les fondements de la pensée économique [1983] de Pribram sont encore moins bien lotis avec une seule référence (voir t. II, 465). De même, A History of Economic Theory and Method [1983] d'Ekelund \& Hebert n'est cité que dans une seule étude (t. I, p. 540, 548).
} 
des éditeurs, sont le fruit de recherches élaborées avec le souci d'éviter les deux écueils interprétatifs bien connus, mais si souvent commis, que constituent les lectures relativistes et rétrospectives.

Au regard d'un ouvrage aussi riche que la Nouvelle histoire..., la place qui nous est impartie nous empêche d'en faire un résumé exhaustif et équilibré. C'est pourquoi nous nous efforcerons d'insister sur les aspects les plus originaux (ou les plus critiquables) de ce livre, tout en donnant, dans la mesure du possible, une idée générale de son contenu.

Le tome I, paru en 1992 mais réédité à l'occasion de la sortie, en 2000, des deux autres tomes, s'étend « des scolastiques aux classiques ». Bien sûr, « le nombre de pages » de l'ouvrage «n'est pas indéfiniment extensible » (t. I, p. 11). Cela dit, était-ce réellement allonger celui-ci sur un lit de Procuste que de lui adjoindre une rubrique consacrée à la réflexion économique de l'antiquité gréco-romaine ? Cette absence est justifiée par le souhait de ne pas commettre d'anachronismes (ibid., p. 18-19), sans compter le problème quasi insurmontable que pose la traduction de certains mots n'ayant plus cours aujourd'hui (ibid., p. 19). Faccarello conclut: « dans le souci d'éviter les erreurs anciennes ou les interprétations hâtives ou incomplètes, nous avons donc préféré débuter cet ouvrage avec la résurgence occidentale d'Aristote » (ibid.).

On pourra toujours railler une telle modestie intellectuelle. Elle illustre toutefois le soin de ne pas verser dans une interprétation rétrospective des textes anciens : à sa manière, cette absence constitue une excellente leçon méthodologique. Une bibliographie sélective (ibid., p. 21-23) est par ailleurs fournie au lecteur dont la curiosité demeurerait inassouvie. Néanmoins, le même genre de scrupules n'est pas mis en avant afin de justifier, en particulier, la non-évocation de la « réflexion économique » chinoise (VII ${ }^{\mathrm{e}}$ $\mathrm{III}^{\mathrm{e}}$ siècle avant $\mathrm{JC}$ ) et indienne ( $\mathrm{IV}^{\mathrm{e}}$ siècle avant $\left.\mathrm{JC}\right)$. Cela peut sembler regrettable. Cependant, du point de vue de l'histoire des idées, il est vrai que ces deux courants ne présentent pas l'intérêt d'avoir irrigué les pensées économiques ultérieures à l'exception, pour autant que nous le sachions, de celle physiocrate - nous avons à l'esprit les propos élogieux que Quesnay et ses disciples tiendront sur le Despotisme de la Chine [1767] et le modèle religieux confucéen.

En revanche, la lecture de la «Première partie » de la Nouvelle histoire..., intitulée «l'économie entre la religion, le droit et la morale du $\mathrm{XII}^{\mathrm{e}}$ au $\mathrm{XVI}^{\mathrm{e}}$ siècle» révèle à quel point la redécouverte d'Aristote fut essentielle pour la pensée occidentale. L'interprétation de cette redécouverte, rappelle A. Lapidus, est rendue toutefois difficile par la nature de son support textuel, parvenu à travers les commentaires d'Aristote livrés par les philosophes et théologiens arabes et les «traductions en cascade » (ibid., p. 25) - arabes puis latines - de son œuvre. En outre, comme l'intitulé de la « Première partie » le suggère, la préoccupation de ne pas tomber dans l'ornière d'une analyse rétrospective est aussi mise en 
avant par Lapidus. À cette aune, sont ainsi envisagés les embryons d'un discours économique consacré à l'usure, à la nature et aux fonctions de la monnaie et, bien sûr, au juste prix. On remarque cependant que Lapidus n'hésite pas à recourir à une terminologie, dont il ne juge pas nécessaire de préciser qu'elle est empruntée à la théorie économique moderne, lorsqu'il envisage les réflexions scolastiques relatives au prêt à intérêt et à ses substituts, situations dans lesquelles le moraliste («principal») doit inciter le créancier («l'agent») à révéler «l'information» dont il dispose, celle-ci renvoyant par exemple au « coût d'opportunité » qu'il supporte (ibid., p. 56 et suiv.). Quoi qu'il en soit, cet usage se trouve replacé dans le cadre d'une argumentation, exempte de tout anachronisme, où est rappelée l'appréhension holiste et finalisée de la société qui gouverne l'esprit scolastique.

C'est au demeurant du point de vue de la communauté dans son ensemble, signale Lapidus, que commença à poindre l'idée, au début du $\mathrm{XV}^{\mathrm{e}}$ siècle, que «l'absence complète de toute opération de crédit provoquerait [...] la misère et le vice » (ibid., p. 66). Alors même qu'elle s'inscrit directement dans le sillage du thomisme, l'école de Salamanque, au siècle suivant, va précisément avoir pour tâche la reconnaissance de la légitimité des prêts à intérêt, sans cesse plus nombreux et plus importants, réalisés entre les marchands. De façon plus large, c'est la panoplie entière de l'activité de ces derniers (comprenant également les gains issus du change des monnaies ou de l'échange de biens) que des écrivains tels que Mercado, Soto et Vitoria vont tenter de concilier avec la doctrine de l'Église. Il s'agit là, estime R. Tortajada de façon légèrement caricaturale, de « l'ultime effort pour intégrer la dimension économique dans un schéma fondamentalement religieux » (ibid., p. 80). L'esprit de la Réforme, s'il s'oppose à la perspective d'ensemble de la philosophie scolastique n'en est pas moins confronté, à l'instar de l'école de Salamanque, à un univers économique en transformation. Et « en pratique » (ibid., p. 84), les réformateurs parviennent à une conclusion similaire à celle de leurs prédécesseurs espagnols : « un taux d'usure modéré est socialement acceptable et compatible avec les principes religieux, surtout s'il s'agit de prêts aux affaires » (ibid.). Enfin, pour les théoriciens du droit naturel, «l'histoire, dans une très large mesure, avait tranché : elle [l'usure] ne présentait plus un véritable enjeu » (ibid., p. 88). Et, si tant est qu'elle soit évoquée, cette notion d'usure fut du reste explicitement dénigrée au profit de celle d'intérêt, en particulier par Grotius.

$\mathrm{Au}$ terme de la lecture de la «Première partie » de la Nouvelle histoire..., un sentiment prévaut : celui de l'existence d'écrivains qui, par delà leurs divergences, sont confrontés à partir du $\mathrm{XV}^{\mathrm{e}}$ siècle à l'expansion de l'activité marchande qu'ils essayent de conceptualiser mais dont ils tendent aussi à prendre, par là même, acte. D'un contexte économique qui livre un objet d'étude, un glissement progressif s'opère ainsi, chez un nombre considérable d'auteurs, vers une reconnaissance, une acceptation puis une légitimation de cet objet. S'il s'agit là d'analyse relativiste, sa crédibilité nous paraît devoir la délester de toute connotation péjorative. 
La « Deuxième partie » de la Nouvelle histoire..., intitulée « Marchands et princes : les auteurs dits mercantilistes ", conforte à certains égards ce dernier point de vue. L'entreprise de justification de la recherche du gain est commune, indique P. Steiner, à des auteurs tels que Thomas Culpeper père, Montchrétien, Mun ou encore Smythe. Mais s'il en résulte une défense du « libéralisme » économique, celle-ci s'avère au service de la bourgeoisie marchande: tant au niveau national qu'international, la perspective d'une harmonie des intérêts économiques est absente du discours mercantiliste - sans compter que la thèse de la liberté du commerce n'est alors pas étayée par une quelconque analyse du système de prix. La pression que l'activité d'une classe sociale particulière peut exercer sur le discours économique est par ailleurs sollicitée par Steiner lorsqu'il évoque, non sans nuances il est vrai, la coïncidence entre la place grandissante accordée à la notion de «balance-travail» et la montée en puissance du secteur manufacturier. De même, Steiner souligne le rôle joué par les marchands interlopes dans la remise en cause des compagnies à privilège et des recommandations protectionnistes véhiculées par la doctrine de la « balance du commerce».

La «Troisième partie» de la Nouvelle histoire..., «Les économies politiques des Lumières: l'affirmation de la pensée libérale », en est peut-être la plus audacieuse et la plus novatrice. G. Faccarello indique que l'objectif est ici d'entériner le déplacement, effectué «à partir des dernières années du XVII ${ }^{\mathrm{e}}$ siècle et pour une très large partie du XVIII ${ }^{\mathrm{e}}$ » (ibid., p. 144), de la pensée économique sur le Continent. La Nouvelle Histoire... se focalise sur le cas de la France mais Faccarello n'omet pas de mentionner l'importance des contributions allemandes, autrichiennes, espagnoles, portugaises et bien sûr italiennes. Toutefois, les références bibliographiques (ibid., p. 151-152) concernant cette pensée économique occidentale ne sont pas légion et révèlent à quel point ce domaine de la recherche a été peu exploré jusqu'à maintenant. Ce n'est pas le moindre mérite de cette «Troisième partie » de la Nouvelle histoire... que de contribuer à dissiper cette forme d' "anatopisme », si l'on ose dire. Mais une telle entreprise est aussi affaire de territoire disciplinaire : Faccarello insiste sur l'emprise qu'exercent encore, à cette époque, «la philosophie politique et la philosophie morale» (ibid. p. 145) sur la pensée économique, cela afin de tempérer l'idée d'une « autonomisation » (ibid.) de cette dernière. Les résultats les plus marquants de cette «Troisième partie» sont ainsi de deux sortes. Premièrement, ceux-ci résident dans la nature continentale des figures intellectuelles qu'ils font tout simplement apparaître ou qu'ils mettent en valeur ; deuxièmement, ces résultats dérivent de l'accent placé sur l'ancrage philosophique de tout ou partie de la pensée économique de plusieurs auteurs - ces deux éléments n'étant bien évidemment pas contradictoires.

Concernant le premier point, le cas de l'infortuné Law peut être évoqué. Le cuisant échec de ses innovations financières en France a terni considérablement sa réputation de théoricien. Or celle-ci mériterait d'être réévaluée, estime A. Murphy, à un point tel que Law devrait paradoxalement « être considéré à la fois comme un "précurseur" du monétarisme ainsi que du keynésianisme » (ibid., p. 176). 
Du monétarisme car, en particulier, «Law est le premier auteur à utiliser l'expression de "demande de monnaie" » (ibid., p. 181). Non vérifiable, ce genre d'affirmation peut toujours être sujet à caution. Admettons du moins que Law a eu le mérite de conceptualiser la notion de « demande de monnaie » et par là même celui de la populariser, à défaut de l'inventer. Par ailleurs, la préfiguration de la pensée keynésienne que Murphy décèle chez Law dériverait de «l'identification des causes du chômage et de la sous-utilisation des ressources dans une offre inadaptée de monnaie et dans un niveau trop élevé du taux d'intérêt » (ibid., p. 182). Cette proposition, qui consiste simplement à donner de la crise une explication de nature monétaire, ne nous paraît pas nécessairement faire de Law un précurseur de Keynes, sans même invoquer le peu de marge de manœuvre que ce dernier accorde à la politique monétaire.

A l'instar de Law, le groupe de Vincent de Gournay se voit également réserver une place à part au sein de la Nouvelle Histoire... La contribution de Murphy, à cet égard, vise l'essentiel : démontrer que l'adhésion de ce groupe aux préceptes « libéraux » ne fut pas si entière que ce que Dupont de Nemours ou Turgot ont bien voulu nous le laisser croire.

Si l'on peut louer le fait qu'une étude soit consacrée à Law et au groupe de Gournay, il n'y a en revanche rien de remarquable, a priori, à ce que l'évocation de la pensée de Turgot occupe une rubrique particulière dans la Nouvelle Histoire... : la démarcation de ce dernier vis-à-vis de Quesnay et des physiocrates est aujourd'hui amplement reconnue. Néanmoins, la spécificité du commentaire de Faccarello est de présenter Turgot, lui-même, comme le chef de file d'une école de pensée dont Condorcet et Roederer seraient les principaux disciples. Dans le sillage de Turgot, sont ainsi mises en valeur les réflexions du premier en matière de finances publiques et celles du second s'agissant de la théorie du capital.

Simultanément, en inscrivant Turgot et ses partisans au sein de l'économie politique dite sensualiste, Faccarello insiste sur les conceptions philosophiques qui sous-tendent leur réflexion économique, deuxième élément qui singularise la plupart des études relatives aux auteurs de la période. Outre celle consacrée à l'école de Turgot, il en va ainsi de l'analyse de la pensée économique de Boisguilbert dans la mesure où elle met l'accent sur l'influence janséniste qui s'y manifeste.

De même, l'intérêt principal du commentaire de P. Steiner sur Quesnay et les physiocrates réside dans la portée qu'il accorde à la distinction philosophique opérée par Quesnay entre la «"liberté animale" (la simple raison) » et la « "liberté d'intelligence" (la raison éclairée) » (ibid., p. 228). Apanage de l'être humain, cette seconde espèce de liberté est constitutive du versant «normatif» (ibid.) du «sensualisme» de Quesnay; elle permet d'envisager ses travaux les plus dogmatiques comme une description de l'idéal comportemental que les agents économiques doivent atteindre, lequel idéal consiste dans l'exercice de l'intérêt bien entendu associé à cette liberté d'intelligence. Il est dès lors possible d'expliquer pourquoi Quesnay n'accorde que peu de place dans son œuvre à l'étude de la conduite effective des agents économiques, celle-ci reposant sur l'exercice de la liberté animale. 
Sur ce dernier point, il est incontestable que la réflexion d'un auteur tel que Smith entend se concentrer en revanche sur une appréciation «positive » du comportement qui, de quelque nature qu'il soit, s'établit en fonction du regard selon lequel autrui l'estime. C'est par un rappel de cette conception que s'ouvre l'étude de l'œuvre du Professeur de Glasgow introduisant la «Quatrième Partie » de l'ouvrage, consacrée aux «économies classiques ». À ce titre, Béraud envisage la pensée de Smith à l'aune de ses réflexions économiques mais aussi de sa philosophie morale, telle qu'elle s'exprime principalement dans la Théorie des sentiments moraux [1759, 1790]. La conciliation entre ces deux perspectives est aujourd'hui admise par tout commentateur avisé de l'œuvre de Smith. Mais ce désir d'accorder un crédit de cohérence à la pensée de Smith se retrouve dans l'exposé de Béraud consacré à des thèmes plus particuliers, tels que la théorie des prix, l'analyse de la rente et l'épineuse question de l'étalon invariable des valeurs. La tentative de présenter la pensée de Smith sous un jour harmonieux s'accompagne également d'un essai de réhabilitation de sa pensée monétaire, dont certains aspects préfigurent la théorie de la Banking School.

Si la lecture du chapitre consacré à Smith ne présente pas de réelles difficultés, il n'en est pas de même de celui consacré aux classiques de la « seconde génération ». Dans sa majeure partie, ce chapitre confronte de façon quasi simultanée la pensée de Malthus, de Ricardo, de Say et d'une bonne dizaine d'autres économistes, cela au prix de digressions - concernant même la littérature secondaire - et surtout de répétitions qui peuvent paraître fastidieuses. Néanmoins, ce désordre formel n'est, somme toute, que le reflet du bouillonnement, de l'ardeur et de la complexité des débats de l'époque. Aussi, le mode d'exposition privilégié par Béraud est-il préférable à celui consistant à présenter les auteurs les uns après les autres, comme le laissait déjà entendre W. Eltis (1993), dans sa propre recension du tome I de la Nouvelle histoire... À ce sujet, tout en rappelant dans son introduction qu'il convient de se défier d'interpréter les résultats analytiques d'une période à la lumière d'un contexte économique qui viendrait les conditionner, Béraud choisit assez naturellement de structurer son commentaire autour des principaux objets d'étude dictés par ce même contexte. Trois questions, liées entre elles, sont retenues, les deux dernières étant subdivisées en plusieurs thèmes. La première de ces questions concerne la population, la seconde porte sur les rendements agricoles et, enfin, la troisième traite de la notion de crise économique. Par ailleurs, dans le corps même de son analyse, Béraud agence parfois son propos en s'appuyant sur le fait que, par delà les divergences qui opposent les auteurs de la période - sur des thèmes tels que la valeur et son étalon, les prix, les variables de répartition, l'épargne... - et des influences qu'ils exercent les uns sur les autres - le cas de Malthus et de Ricardo est, on le sait, le plus exemplaire-leurs positions s'articulent fréquemment autour d'une même référence intellectuelle, à partir de laquelle chacun construit ses propres spéculations : la Richesse des Nations de Smith.

La contribution de Béraud fait quasiment l'impasse sur les débats monétaires. Pourtant, Béraud reconnaît en introduction, que « la controverse la plus longue et la plus passionnée fut celle suscitée par 
les questions monétaires » (ibid., p. 369). Il est à ce titre regrettable que Béraud ne prenne pas la peine de renvoyer à l'étude de De Boyer consacrée à de telles questions. En outre, quoique cette étude s'étende jusqu'à la réforme du système bancaire anglais de 1844, elle apparaît relativement mal positionnée dans le tome I puisqu'elle figure à la suite du chapitre que R. Arena et d'A. Maricic consacrent à « la postérité ricardienne» et à «l'œuvre de J. S. Mill». Est-ce parce que les éditeurs ont voulu préserver une dichotomie entre « analyse réelle » et « analyse monétaire » dans l'économie de l'ouvrage ? En tout état de cause, cette séparation contrarie la perspective chronologique qui domine celui-ci.

On sait que certaines œuvres en occultent d'autres. Il en va ainsi de celle de Smith qui escamota celle de Steuart, de celle de Ricardo qui fit de l'ombre à celle de Thornton. De même, comme le rappelle Faccarello (t. II, p. 10), la pensée de Marx a longtemps éclipsé celle des « réformateurs sociaux » du début du $\mathrm{XIX}^{\mathrm{e}}$ siècle. Il est à cet égard appréciable que le tome II de la Nouvelle Histoire..., s'étendant «des premiers mouvements socialistes aux néo-classiques», s'ouvre par un chapitre spécifiquement consacré à ces auteurs - essentiellement Hodgskin, Owen, Proudhon, Saint-Simon, Sismondi. On relèvera cependant que R. Ege n'hésite pas à émailler son commentaire, lorsqu'il évoque la pensée d'Owen et de Proudhon, de critiques similaires à celles formulées par Marx, qu'il cite du reste incidemment. Concernant par exemple Owen, Ege centre sa critique sur l'aspect contradictoire que revêt le souhait de vouloir construire une communauté et un homme nouveaux à partir et au sein de référents eux-mêmes inscrits dans une société déjà existante : la société capitaliste. L'analyse d'Ege ne fait ainsi que reproduire peu ou prou celle, fameuse, figurant dans le Manifeste communiste [1848]. Notons au passage que dans ce dernier texte, Marx épinglait aussi la figure intellectuelle de Fourier, dont il est dommage qu'elle soit ignorée non seulement par Ege mais également par l'ensemble des auteurs ayant contribué à la Nouvelle histoire... ${ }^{6}$ Au total, la réhabilitation par Ege des auteurs de la période n'est vraiment effective qu'à propos de Sismondi, qualifié de «meilleur économiste» (t. II, p. 30) de son temps. L'analyse de la crise livrée par ce dernier est alors étudiée en détails. Il est toutefois regrettable qu'Ege ne fasse aucun renvoi aux commentaires d'Arena et de Béraud formulés à ce sujet dans le tome I, même s'il est tout à fait légitime d'envisager Sismondi comme un auteur appartenant à plusieurs traditions (ricardienne, post-ricardienne, réformatrice, socialiste), ce qui justifierait l'exposé de son analyse à plusieurs endroits dans un même ouvrage d'histoire de la pensée économique.

Plutôt que de s'en tenir, dans un souci pédagogique et simplificateur, à un exposé élémentaire des principales idées de Marx, Faccarello essaye, dans le chapitre suivant, de dégager les aspects antagoniques qui animeraient le projet intellectuel de l'auteur du Capital. Cela à un point tel que Marx est l'auteur d'envergure dont la clarté et la cohérence des analyses sont, dans la Nouvelle Histoire..., les plus mises à l'épreuve. Seulement, la tension du commentaire vers ce dernier objectif se fait

\footnotetext{
${ }^{6}$ À l'exception d'A. Cot (t. I, p. 299) lorsqu'elle traite de la postérité intellectuelle de Bentham.
} 
naturellement au prix de la négligence d'un certain nombre de thèmes reconnus habituellement comme relevant du marxisme, quelle que soit par ailleurs la dose de singularité que Marx leur insuffle. Il en va ainsi de la question de l'aliénation, de la définition du mode de production, de la conception matérialiste de l'histoire ou encore de la lutte des classes. Certes, on devine que Faccarello ne juge pas ces questions anecdotiques, il lui arrive même de solliciter certaines d'entre elles mais, par dessus tout, on a le sentiment qu'il les suppose connues du lecteur. Dès lors son commentaire s'adresse véritablement à des spécialistes et, par là même, fait montre d'un recul critique prononcé.

Ce recul critique réside dans l'idée selon laquelle «la construction marxienne repose sur trois exigences » (ibid., p. 76), chacune d'entre elles ayant la particularité de «mett[re] en cuvre - sous des appellations identiques - des définitions différentes, et incompatibles entre elles, des principaux concepts : ceux de valeur et de monnaie en particulier » (ibid., p. 78). Dans le même mouvement, Faccarello fait apparaître une emprise hégélienne dans les écrits de Marx qui ne se cantonne pas à celle relevant de la philosophie de l'histoire et il cherche ainsi à montrer qu'il existe une «médiation constante, chez lui [Marx], de l'économie politique, du moins de ses concepts fondamentaux, par le legs hégélien » (ibid., p. 83).

Le premier impératif qui anime le projet de Marx consiste à montrer la relativité historique du mode de production capitaliste et de ses lois de fonctionnement. Dans cette optique, la théorie de la valeurtravail est jugée centrale et Faccarello explique que le désir de Marx de déterminer la « substance » de la valeur relèverait d'une démarche empruntée à Hegel. Cette influence de Hegel sur Marx apparaît cependant peu dans le cadre de la présentation de cette première « exigence »; laquelle repose, indique Faccarello, sur « une analyse qui se déroule fondamentalement en termes réels » (ibid., p. 84), cela à partir du moment où Marx définit justement la substance de la valeur a priori en termes de travail. L'analyse de Marx est alors située dans la perspective classique des prix naturels, où la monnaie n'exerce qu'un rôle de numéraire. Dominante dans l'œuvre de Marx, la première " exigence » côtoie, déclare Faccarello, celle consistant à mettre en exergue la spécificité du mode de production capitaliste par rapport aux autres modes de production. Seulement, cette spécificité repose inévitablement sur la mise au premier plan du rôle de la monnaie, justement déconsidéré dans le cadre de la première approche. En outre, la notion de prix de marché se trouve elle-même réévaluée par rapport à celle de prix naturel car un produit doit être vendu, c'est-à-dire à proprement parler être reconnu comme marchandise, pour que le travail qu'il contient soit lui-même validé socialement-cette idée d'une reconnaissance sociale du travail se trouvant aussi chez Hegel. La troisième exigence sur laquelle se construit l'œuvre de Marx, estime Faccarello, renvoie à la nature de la société communiste succédant à celle capitaliste, bien que Marx ait été - cela est bien connu - relativement circonspect en la matière. Puisque l'abolition de l'échange marchand caractérise la société communiste, Marx doit alors montrer a contrario qu'une économie capitaliste est nécessairement une économie monétaire, tâche qu'il entreprend en fondant son 
argumentation sur ce que Faccarello nomme "une déduction dialectique des concepts» (ibid.). L'objectif de Marx serait, dans cette optique, de partir de l'opposition entre valeur et valeur d'usage dont Faccarello tente de montrer qu'elle est «purement formelle » (ibid., p. 123) - pour en déduire successivement, en s'inspirant de la dialectique hégélienne, le concept de monnaie, celui de capital et, enfin, celui de travail salarié. Monnaie, capital et exploitation se trouvent ainsi intimement et exclusivement reliés, conformément à la fin que Marx se serait fixée.

D’une certaine façon, les aspects conflictuels présents dans la pensée de Marx seront relayés par «les controverses autour du Capital» auxquelles sont consacrées les deux chapitres suivants de la Nouvelle Histoire... Cela semble particulièrement vrai pour « les débats autour de la loi de la valeur», chapitre dans lequel Faccarello retrace notamment l'histoire du problème de la transformation des valeurs en prix, depuis dès avant la parution du Livre III, jusqu'à sa solution définitive dans les années 1960-1970. On sait, à ce sujet, qu'une solution générale au problème de la transformation s'est dégagée à partir du moment où Sraffa a résolu le problème de l'étalon de valeur de Ricardo, dans le cadre du marxisme dit matriciel. Or, le fait que la question de l'étalon de valeur de Ricardo et celle de la transformation aient été résolues simultanément, en utilisant d'ailleurs les mêmes instruments mathématiques - à savoir les vecteurs propres d'une application linéaire - indique selon nous que la problématique de Marx sur la valeur ne faisait que déplacer les difficultés que posait celle de Ricardo. Pour ce qui concerne « les crises », second chapitre relatif aux « controverses autour du Capital», C. Tutin souligne le rôle variable accordé aux deux pistes suggérées par Marx, celle de la baisse tendancielle du taux de profit et celle «d'une incompatibilité entre les conditions de la croissance et les contraintes de la répartition » (ibid., p. 202).

Le principal résultat de ces deux chapitres est qu' « un siècle de débats a conduit à invalider une partie des thèses de Marx » (ibid., p. 228), à savoir, d'une part, celle concernant la transformation et, d'autre part, celle renvoyant à l'évolution tendancielle du taux de profit - thèmes dont Faccarello avait déjà souligné les incohérences dans son étude consacrée à Marx lui-même. Cela dit, les débats relatifs à la reproduction, analysés par C. Tutin, « ont fait ressortir l'intérêt des intuitions de Marx » (ibid., p. 228) qui « apparaît comme un véritable pionnier » (ibid., p. 226), en anticipant sur les théories modernes de la croissance. Comme le soulignent aussi bien Faccarello que Tutin, il demeure que ces controverses sont d'autant plus difficiles à analyser que les propos des différents auteurs sont, en la matière, souvent brouillés par leur propre conscience politique, considération que Chavance met également en avant dans son analyse de « la théorie de l'économie socialiste».

De ce point de vue, le commentaire des thèses développées par les marginalistes et les néoclassiques apparaît, dans une certaine mesure, moins problématique. La «Sixième partie» de la Nouvelle histoire... qui leur est consacrée peut être décomposée selon trois volets. 
Un premier volet, assez succinct, est consacré aux « origines de la pensée néoclassique » que F. Etner fait remonter à D. Bernoulli, Cournot, Dupuit, Gossen et Thünen. Cette contribution, qui se concentre sur le thème de la demande, de l'utilité et de la productivité marginales, aurait peut-être gagnée à être replacée dans le cadre d'une interrogation plus vaste sur les causes de la « révolution marginaliste ». Outre le développement du raisonnement à la marge lui-même, aurait alors été notamment discutée la pertinence d'une explication fondée sur la désagrégation progressive de l'édifice ricardien, orchestrée par ceux-là même qui se présentaient comme les disciples de Ricardo: les commentaires d'Arena au sujet de J. Mill et McCulloch et surtout ceux de Maricic à l'endroit de J. S. Mill, figurant à la fin du tome I de la Nouvelle histoire..., auraient de la sorte trouvé un riche écho.

Le second volet de cette «Sixième partie » rend compte de la naissance, en tant que telle, des différents courants du marginalisme, puis de leurs évolutions ultérieures. Axé sur un commentaire «par auteurs » plutôt que " par thèmes », ce second volet est particulièrement approfondi et nuancé. Il s'ouvre par une étude de Béraud concernant les autrichiens, à savoir Menger puis Böhm-Bawerk et Wieser, laquelle étude met à la fois en lumière la spécificité de ce courant - caractérisée en particulier par un « rejet de l'économie mathématique» (ibid., p. 297) et «le rôle du savoir dans les décisions économiques » (ibid., p. 298) - et la diversité des approches entre ses différents auteurs - quoique non complètement contradictoires, les solutions proposées au fameux problème de l'imputation en témoignent.

Ce souci de ne pas envisager chacun des courants représentatifs du marginalisme comme un bloc homogène se retrouve, il n'y a là rien de surprenant, dans la présentation de « Jevons, Marshall et Edgeworth: les néoclassiques anglais », également effectuée par Béraud. Il faut ajouter que sont analysées les contributions de plusieurs autres auteurs, tant contemporains des précédents (Berry, Wicksteed) que plus récents (Fisher, Viner, Frisch). À l'occasion d'un commentaire assez largement rétrospectif, Edgeworth, par sa croyance en l'indétermination de l'équilibre lorsque le nombre d'échangistes est fini, est présenté comme un précurseur, de son temps incompris - si l'on excepte, dans une certaine mesure, de Pareto (auquel Steiner consacre par ailleurs une étude spécifique). Jevons, qui quant à lui estime que « l'équilibre est déterminé, même si le nombre d'agents présents sur le marché est [...] fini ou, même, petit » (ibid., p. 398), est envisagé par Béraud d'un œil plus sévère ; le décalage entre son analyse économique et la transcription mathématique de celle-ci, assez frustre, est à cette occasion souligné. S'agissant de Marshall, Béraud rappelle que la conception de l'équilibre à long terme reste proche des classiques : les échanges à «faux prix », correspondant à un mécanisme de gravitation, ne modifient pas l'équilibre final, sous l'hypothèse de la constance de l'utilité marginale de la monnaie. Certes, le commentaire de Béraud fait apparaître les aspects novateurs de la pensée du professeur de Cambridge, cependant, la tonalité générale est assez critique à l'encontre de ce dernier, à propos de l'équilibre partiel et sur la façon de concilier les rendements d'échelle et la concurrence. 
Walras, enfin, dernier grand représentant du marginalisme, bénéficie d'un traitement privilégié. Dans le chapitre qui lui est spécifiquement consacré, J. Lallement mentionne qu'à la différence des «autres fondateurs du marginalisme, [...] Walras développe une vision globale de l'économie elle-même insérée dans une représentation complète et explicite de la société » (ibid., p. 449). Lors de l'exposé de la partie de son projet intellectuel que Walras conduit le plus loin, l'économie pure, une comparaison avec la pensée de Menger eût peut-être été judicieuse : à partir de positions philosophiques proches néo-kantiennes ou kantiennes -, Walras développe une analyse qui s'oppose au subjectivisme de Menger et sa théorie symétrique de la valeur fait dépendre le prix de la demande et de l'offre. Lallement souligne par ailleurs que l'offre étant chez Walras issue du modèle simple à deux marchandises où le prix est exprimé à l'aide de l'autre marchandise, l'effet de richesse se traduit à partir d'un certain point par une offre décroissante et les équilibres multiples n'ont rien d'exceptionnel. Au total, et en dépit d'un reproche fait à son manque de clarté terminologique au sujet de la valeur d'échange et de la rareté, Walras se voit crédité de nombreuses avancées : «formulation la plus claire qui soit de la problématique néoclassique » (ibid., p. 453); «nouveauté de l'argumentation mathématique » (ibid., p. 464); « richesse [...] [d'une] analyse [...] qui introduit des problèmes et des concepts nouveaux et féconds » (ibid., p. 494).

Cette image positive accordée à Walras est renforcée par la lecture du troisième et dernier volet de la « Sixième partie » de la Nouvelle histoire... De nature thématique, ce volet tend en effet à présenter la construction de Walras comme la bonne réponse aux bonnes questions, là où nombre d'autres économistes seraient restés dans l'approximation. D’une part, concernant la théorie du capital, A. Rebeyrol mentionne que «c'est a priori le modèle de Walras qui doit être pris comme référence de la théorie néoclassique » (ibid., p. 568), cela parce que Walras envisage clairement le capital comme un ensemble de biens hétérogènes. D'autre part, la contribution de De Boyer portant sur la monnaie - thème qui clôturait également le tome I - reconnaît à Walras le mérite d'avoir accordé à celle-ci un traitement qui ne relève pas de la dichotomie et qui n'est pas entaché de circularité ; ceci, bien sûr, dans le cadre de l'équilibre général dont le statut très particulier a été souligné par plusieurs des contributions précédentes, auxquelles des renvois auraient été bienvenus.

Le troisième tome de la Nouvelle histoire... s'étend «Des institutionnalistes à la période contemporaine ». La partie qui ouvre ce tome traite plus précisément de la sociologie économique de Simiand, de l'école historique allemande et, enfin, de l'institutionnalisme de Veblen et de ses successeurs. La raison d'être de cette partie réside dans la position critique, historiquement dominante, que ces économistes adoptent à des degrés divers vis-à-vis des pensées classiques et néoclassiques. Ce regroupement, s'il est justifié, ne va pas sans poser problème quant à la situation, au sein de la Nouvelle histoire..., du commentaire consacré à certains auteurs de l'école historique allemande. Pour des raisons 
de chronologie d'abord. Hildebrand et Rosher sont nés un peu avant Marx, tandis que Knies n'était son cadet que de trois ans. Pour des raisons de fond ensuite. Comme Marx, les auteurs de la première et surtout de la seconde école historique allemande insistent sur la relativité des lois de l'économie. En outre, Béraud, dans le commentaire qu'il consacre aux autrichiens, n'avait pas manqué de mentionner que « la tradition dont il [Menger] hérite est celle de [...] Rau, Roscher, Knies » (t. II, p. 294) et que la querelle des méthodes joue un rôle important dans la cristallisation du subjectivisme autrichien. Signalons enfin que l'influence de l'école historique allemande est très présente chez Marshall, dont les principales analyses sont essentiellement commentées dans le tome II de la Nouvelle histoire... Toutefois, de nombreuses filiations - reconnues ou rejetées - et la continuité qui existent entre les divers membres de cette école sont soulignées de façon extrêmement précise par V. Gioia, dont la richesse du commentaire ne pourra que réjouir le lecteur francophone.

Contrairement à certains auteurs de la « Septième partie » de la Nouvelle histoire..., dont la place et l'identité, on l'a vu, posent problème, ceux évoqués à l'occasion de la « Huitième partie » se situent de plein pied au XX $X^{\mathrm{e}}$ siècle. Comme l'indique Béraud (ibid., p. 119), l'intitulé de l'ouvrage de Shackle, Les Années de haute théorie, est l'éponyme de cette partie, s'étendant de la Première à la Seconde Guerre Mondiale. Un panorama assez vaste des auteurs ayant contribué aux modélisations de la demande (Hallen \& Hicks, Samuelson, Slutsky...) et des marchés (Chamberlin, Kalecki, Knight, Stackelberg...) est tout d'abord dressé par M. Rainelli qui souligne aussi le lien entre la question des rendements d'échelle et le schéma d'offre de Sraffa. Son étude est relayée par celle d'A. Zylberberg, consacrée aux économistes ayant travaillé sur l'équilibre général et, en particulier, sur la question de son existence (Von Neumann, Wald...) et de sa présentation dans un cadre dynamique (Lindahl, Myrdall et surtout Hicks). Le chapitre rédigé par C. Gehrke et H. D. Kurz, intitulé « Le développement de la macroéconomie dans l'entre-deux-guerres : l'apport continental» revient sur l'apport de l'école suédoise et relate la controverse entre Keynes et Hayek avant d'envisager d'autres contributions sur la théorie du cycle. Si cette étude évoque ainsi, comme celle qui la précède, les travaux de Wicksell, Lindhal et Myrdal, elle est néanmoins plus explicite sur les processus cumulatifs de Wicksell ou sur l'équilibre temporaire. Elle énonce en outre clairement ce qui demeure le véritable projet de l'école suédoise : « construire un pont entre la théorie de la monnaie et la théorie de l'équilibre général » (ibid., p. 195) et elle rappelle que les économistes suédois prônèrent, avec un certain succès d'ailleurs, des politiques de type keynésien.

Une nouvelle analyse de la pensée de l'école suédoise intervient à la fin de cette «Huitième partie », à l'occasion de l'étude que C. Montet consacre à la théorie des relations économiques internationales. Au sujet de la théorie pure du commerce international, Montet rappelle en effet que l'entre-deux-guerres est la période au cours de laquelle l'approche classique découlant directement de l'avantage comparatif de Ricardo, cède le pas au modèle néoclassique, mis en avant par Heckscher et 
Ohlin. C'est aussi l'époque où se développent une approche normative de la politique commerciale et l'étude de son influence sur la répartition du revenu national. C'est enfin celle, ajoute Montet, où apparaissent les premières études qui remettent en cause l'hypothèse de concurrence parfaite. La triple influence des bouleversements monétaires, du problème des réparations allemandes et de la révolution keynésienne suscite un renouveau de la théorie monétaire internationale. Le point de vue de Keynes, d'après qui «l'Allemagne va devoir porter un double fardeau : le montant des réparations et une détérioration de ses termes de l'échange » (ibid., p. 324), est combattu par Ohlin : ce dernier reproche à Keynes de sous-estimer l'effet du paiement des réparations sur la demande étrangère d'une part, et sur le revenu intérieur, d'autre part. En fait, même si la démonstration d'Ohlin « manque de rigueur » (ibid., p. 325) selon Montet, c'est lui qui tient le rôle du keynésien, alors que « Keynes se trouve du côté où l'on ne l'attend guère, celui de la pensée orthodoxe avec Pigou et Taussig » (ibid.).

Qu'elle soit adoptée ou rejetée, la pensée keynésienne occupe ainsi une place importante dans plusieurs commentaires de cette «Huitième partie» de la Nouvelle histoire. L'étude qui lui est spécifiquement consacrée, rédigée par R. Dos Santos Ferreira, constitue le point culminant de cette partie, sinon le centre ou l'aboutissement. Précisons que le choix du commentateur, sans doute le seul possible, est ici de s'en tenir à une analyse de la Théorie générale en tant que telle, au dépens des différentes exégèses ou du reste des écrits de Keynes. Après avoir évoqué le contexte économique et le climat intellectuel dans lesquels a été élaborée l'œuvre économique majeure de Keynes, Dos Santos Ferreira se livre à une lecture relativement linéaire de l'ouvrage - qu'il juge « peu ou mal lu » (ibid., p. 236) -, étude dont nous relèverons seulement les aspects les plus originaux concernant le marché du travail et la demande effective.

L'originalité de l'analyse keynésienne du marché du travail est bien sûr la notion de chômage involontaire dont la définition est rappelée par Dos Santos Ferreira : «Le chômage est involontaire si, à la suite d'un virtuel accroissement de faible ampleur de la valeur [ou plus exactement du prix] du panier de biens salariaux, l'offre et la demande de travail excèdent toutes deux l'emploi courant, à salaire nominal constant» (ibid., p. 249). On pourrait se demander pourquoi Keynes formule cette définition relativement complexe, alors que si l'offre et la demande de travail excèdent l'emploi courant, logiquement, l'emploi augmente. Cela dit, c'est bien ainsi, et à juste titre, que Dos Santos Ferreira interprète la définition du chômage involontaire : « La nature involontaire du chômage est attestée par la disposition des travailleurs, individuellement et collectivement, à accepter de travailler pour un salaire réel réduit » (ibid.). Sur la question de savoir, qui ou quoi, est responsable de ce salaire réel trop élevé, le commentateur nous semble être dans le vrai lorsqu'il se démarque radicalement des interprétations en termes de rigidités nominales. S'appuyant également sur le chapitre XIX de la Théorie générale («Variations des salaires nominaux »), sa lecture insiste sur la rupture qu'opère Keynes par rapport à son professeur Marshall, en passant d'une analyse en termes d'équilibre partiel à une analyse en termes 
d'équilibre, sinon général, du moins global. Dans cette optique, la rigidité des salaires apparaît, non seulement comme le résultat de la réaction normale des travailleurs qui cherchent à défendre leur pouvoir d'achat relatif, mais aussi comme un optimum de second rang qui évite à l'économie de sombrer dans une spirale déflationniste.

En l'absence d'un mécanisme de retour à l'équilibre sur le marché du travail, le niveau d'activité et d'emploi dépend du principe de la demande effective qui, comme le souligne Dos Santos Ferreira, s'inscrit dans un cadre plus marshallien. Se pose, alors, un problème d'agrégation, d'autant que les anticipations concernent chacun des marchés et sont celles de chacune des entreprises. Quoi qu'il en soit, le revenu ou l'emploi est déterminé en dehors du marché du travail et peut correspondre à un chômage involontaire qui traduit un défaut de coordination des choix intertemporels. Keynes rejette l'interprétation en termes de variables ex-ante et ex-post car l'équilibre de court terme est réalisé quel que soit l'état des anticipations à long terme. En définitive, l'absence ou le défaut de coordination repose sur la distinction entre les anticipations à court terme et les anticipations à long terme, qui « renvoie à la distinction marshallienne entre courte et longue périodes » (ibid., p. 263). Le lien entre le présent et le futur repose sur la monnaie et les marchés financiers. L'interprétation selon laquelle ces derniers n'assureraient pas cette fonction de coordination parce que «les flux d'entrée et de sortie du marché, c'est-à-dire de création et d'extinction des titres, peuvent être négligeables relativement aux stocks d'actifs existants, prêts à passer de main en main » (ibid., p. 270), demande d'après nous à être maniée avec précaution. C'est en effet la demande nette qui gouverne l'évolution des cours des actifs financiers, cours des actifs financiers qui joue un rôle comparable au prix des biens capitaux dans le Traité sur la monnaie. Cependant, c'est parce que le lien entre le présent et le futur repose aussi sur la monnaie que les marchés financiers, aux yeux de Keynes, s'avèrent incapables de réaliser une telle coordination, compte tenu de leur caractère non seulement spéculatif mais surtout spéculaire.

Faire l'histoire de la période récente représente une gageure, mais le défi est sans doute encore plus difficile à relever s'agissant de l'histoire de la pensée. C'est néanmoins celui que se donne la dernière partie qui s'organise autour de cinq thèmes principaux que nous évoquerons successivement : la méthodologie, l'équilibre général, la macroéconomie, les relations économiques internationales et l'économie du développement.

Le chapitre de $\mathrm{P}$. Mongin - « La méthodologie économique au $\mathrm{XX}^{\mathrm{e}}$ siècle. Les controverses en théorie de l'entreprise et la théorie des préférences révélées »-couvre plusieurs domaines, comme l'indique son intitulé. La partie consacrée à la méthodologie oppose l'apriorisme à l'empirisme et fait apparaître une position intermédiaire, issue de J. S. Mill que l'on retrouve chez Robbins. Il est à nouveau regrettable que ces rappels méthodologiques ne soient pas accompagnés d'un renvoi à certaines autres contributions de la Nouvelle Histoire..., ces dernières abordant de façon détaillée les conceptions méthodologiques des auteurs précités. Le deuxième thème abordé est celui de la controverse entre les 
marginalistes et le groupe d'Oxford dont les membres estimaient que l'entreprise ne maximisait pas le profit ; débat qui, évidemment, n'est pas étranger à celui sur les formes de concurrence. Au passage, on signalera que l'exposé de Mongin aurait sans doute gagné en intelligibilité s'il avait été accompagné de graphiques, puisqu'il fait intervenir, de façon littéraire, des notions telles que celle de courbes de coûts moyens et de coûts marginaux, de courte période et de longue période. La dernière question envisagée par Mongin renvoie à la théorie des préférences révélées de Samuelson qui, en quelque sorte, couronne la théorie du comportement du consommateur et boucle le parcours commencé par Pareto, avec l'introduction de l'utilité ordinale.

Les deux contributions suivantes abordent des problèmes plus généraux. Celle que B. Guerrien et C. Pignol consacrent à « la théorie de l'équilibre général depuis 1939 », montre que la démonstration de l'existence de l'équilibre général est un bon exemple de découverte simultanée mais il relativise aussi la portée du résultat. Sur un autre plan, il est rappelé que les efforts vont porter sur l'intégration de la monnaie dans un modèle d'équilibre, de façon à lever l'indétermination de sa valeur dans la théorie néoclassique. Enfin, d'autres travaux envisagent des modèles d'équilibre en concurrence imparfaite, avec comme cas limite l'équilibre à prix fixes. Le chapitre suivant d'A. D'Autume s'intitule « l'essor de la macroéconomie », née selon lui «après Keynes » (ibid., p. 417), même si les précurseurs sont nombreux. Sa genèse se confond, pour D'Autume, avec l'histoire du courant de la synthèse, de Hicks à Patinkin. La dynamisation de la synthèse peut passer par « la courbe de Phillips [qui] fournit alors un moyen d'endogénéiser le salaire nominal » (ibid., p. 423). Mais cette courbe met en lumière le rôle des anticipations et renforce la crédibilité du monétarisme. «De manière à peine caricaturale, on peut considérer que ce modèle macroéconomique standard est keynésien à court terme et monétariste à long terme » (ibid., p. 424). Cette forme de division du travail marque un échec du courant keynésien, la fin de la synthèse et un recul de la macroéconomie tandis que, parallèlement, après les anticipations rationnelles, la nouvelle macroéconomie classique conclut à l'impuissance des politiques économiques. On retrouve dans ce chapitre des thèmes qui avaient déjà été abordés dans l'étude de $\mathrm{B}$. Guerrien et $\mathrm{C}$. Pignol sur l'équilibre général - dans sa version monétaire. Mais sans doute ne faut-il pas s'en étonner, l'équilibre général représentant une sorte de macroéconomie néoclassique.

Rédigé par B. Guillochon, le commentaire consacré aux «échanges internationaux » et aux «ajustements monétaires» prolonge celui de C. Montet relatif à l'entre-deux guerres. Il relate l'évolution des théories de la spécialisation, tant de celle classique qui requiert la programmation linéaire, que de celle néoclassique qui devient alors le modèle Heckscher-Ohlin-Samuelson. Guillochon évoque également les approches alternatives qui justifient la politique commerciale stratégique. Sur les questions monétaires internationales, il signale que l'approche par les revenus révèle, en même temps que leur impact négatif, la possibilité de déséquilibres durables, et donc, aussi, de transferts unilatéraux, confortant ainsi la position d'Ohlin dans la controverse sur les réparations de l'Allemagne. La fin du 
système de Bretton Woods, rappelle Guillochon, donne une actualité nouvelle à la question des taux de change et la thèse optimiste selon laquelle ils seraient déterminés par la parité des pouvoirs d'achat ne semble pas confirmée, à l'inverse de celle pessimiste sur la possibilité de déséquilibres durables.

La Nouvelle histoire... se clôt, enfin, par un chapitre d'E. Assidon consacré à « l'émergence d'un nouveau domaine : l'économie du développement ». Il nous offre d'abord un tableau saisissant et détaillé des différentes politiques de développement, qui apparaissent comme le laboratoire des différents courants théoriques. Hélas pour les théories, mais surtout pour les pays en développement, la plupart de ces voies se sont révélées être des impasses. Dès lors « le temps des théoriciens » est révolu, voici celui « des experts», avec une banalisation de la question du développement, qui rentre dans le champ commun des théories de l'échange international ou de celles de la croissance, voire une mise sous tutelle des pays concernés par les dits experts, qui imposent des politiques d'ajustement structurel.

$\mathrm{Au}$ terme de la lecture de cette «Neuvième partie» de l'ouvrage, on a le sentiment que si le statut des chapitres qui le composent n'est certes pas contestable, il est en revanche regrettable qu'une importance plus grande ne soit pas accordée à des domaines tels que l'économie publique, l'économétrie, la théorie des jeux et la théorie de l'information, ou à certains auteurs - des prix nobels tels que Akerlov, Becker, Buchanan, Haavelmo, Markowitz, North ne sont jamais cités. Néanmoins, cet arbitraire est quasi inévitable face à la richesse et à la diversité de l'analyse économique moderne, laquelle analyse n'est à strictement parler, au demeurant, pas encore «rentrée dans l'histoire » puisqu'elle nous est précisément contemporaine. En outre, Faccarello, dans l'«Introduction » de cette « Neuvième partie» de l'ouvrage, fournit au lecteur un certain nombre de références bibliographiques afin de combler ce que l'on pourrait considérer comme un vide.

On ne saurait trop louer Béraud et Faccarello d'avoir su mener à bien, au terme de presque une décennie, cet effort éditiorial considérable que constitue la publication de la Nouvelle histoire de la Pensée Économique. Cela dit, leurs qualités particulières de direction et de coordination, quoique indéniables, ne peuvent, à elles seules, être invoquées pour rendre compte de l'envergure collective d'un tel ouvrage, dont on rappellera qu'elle est unique dans le domaine de l'histoire de la pensée économique. Cet aspect collectif reflète aussi l'accélération de la spécialisation qui s'est manifestée au sein de la pensée économique, à partir de la seconde moitié du siècle dernier.

Nous entendons par là deux phénomènes. Premièrement, le fait que ce qu'il est d'usage de désigner par le terme de «science économique» se soit peu à peu dispersé entre plusieurs branches, certes plus ou moins ramifiées et liées entre elles, mais ayant chacune leurs chercheurs émérites : citons, entre autres, la microéconomie et la macroéconomie, l'économie du travail, l'économie publique, les 
théories de la croissance, du développement, du commerce international, de la monnaie... À cet égard, il est significatif que sur la trentaine d'auteurs ayant participé à la rédaction de la Nouvelle histoire..., pas moins de la moitié s'est consacrée à l'histoire de la pensée économique du $\mathrm{XX}^{\mathrm{e}}$ siècle. Bref, il est d'autant plus difficile à un seul esprit de développer une approche historique compréhensive de sa discipline que celle-ci apparaît sous un jour non seulement dense, mais aussi éclairé par plusieurs faisceaux non nécessairement convergents. Deuxièmement, le développement accéléré de la pensée économique au lendemain de la Seconde Guerre Mondiale s'est traduit par une reconnaissance progressive de l'intérêt que pouvait représenter l'étude, en tant que telle, de son histoire. Et, en ce domaine, la multiplication des postes d'enseignants et de chercheurs, des revues et des manifestations scientifiques s'est également accompagnée d'une spécialisation accrue.

Ce double phénomène de spécialisation - d'une part de la science économique, d'autre part de son analyse historique - trouve, avec la Nouvelle histoire de la pensée économique, une belle et singulière expression de son éclosion. Or, si l'on tente malgré tout d'attribuer un défaut majeur à cet ouvrage, celui-ci se révèle être la contrepartie presque inévitable de sa qualité principale, celle d'être un produit issu d'une division du travail intellectuel. À l'exception des éditeurs, aucun commentateur n'a visiblement lu, en effet, l'ensemble de la Nouvelle Histoire... De telle sorte que, comme nous avons eu fréquemment l'occasion de le mentionner, il existe certaines redites et surtout peu de renvois entre les contributions $^{7}$. Toutefois, cette insuffisance est en partie comblée par les nombreuses "Introductions » des éditeurs et par la présence d'un système d'index, il est vrai lacunaire par certains aspects ${ }^{8}$. Une deuxième imperfection associée au fait que l'ouvrage a été rédigé à plusieurs mains tient au traitement pour le moins hétérogène de l'élément biographique. En la matière, le manque de coordination est difficilement contestable: les biographies sont de longueur très inégales et, pour certains auteurs, inexistantes ${ }^{9}$. Cette anomalie n'est pas mineure si l'on estime, même si cela demeure discutable, que la vie d'un écrivain est très étroitement liée à son œuvre. Un troisième problème, sans doute plus épineux, consiste dans la disparité critique dont font preuve les commentaires : certaines études ${ }^{10}$ sont assez sévères vis-à-vis des auteurs qu'elles considèrent, tandis que d'autres ${ }^{11}$ se veulent plus neutres et en

\footnotetext{
${ }^{7}$ Ils se situent principalement aux endroits suivants : t. I, p. 193, 195, 254, 268, 274, 281 ; t. II, p. 84, 99, 165, 173, 174, 181, 182, 202, 209, 219, 244, 577 ; t. III, p. 136, 237, 359, 380.

${ }^{8} \mathrm{Il}$ n'existe pas d'index thématique pour les tomes II et III et l'index nominal comporte quelques omissions. Au gré de nos lectures, nous avons relevé les oublis suivants, en ne tenant pas compte des auteurs cités dans les bibliographies: D’Alembert : t. I, p. 148 ; Buchanan, t. I, p. 411 ; J. B. Clark, t. II, p. 526-530 ; Commons, t. III, p. 105 ; Diderot, t. I, p. 318 ; Galbraith, t. III, p. 108 ; Hume, t. II, p. 574, 577 ; Keynes, t. I, p. 176 ; Lauderdale, t. I, p. 411 ; Ross, t. III, p. 76 ; Schumpeter, t. I, p. 204, 411 ; Shubick, t. II, p. 418 ; Sraffa, t. I, p. 428.

${ }^{9}$ On regrettera ainsi l'absence d'indications biographiques concernant les «scolastiques » (Buridan, Duns Scot, Oresme, Thomas d'Aquin...) et les « mercantilistes » (Bodin, Child, Culpeper père et fils, Davenant, Locke, Montchrétien, Mun, Norh, Petty...). Il n'existe pas non plus de biographies d'auteurs aussi fameux que Cournot, Luxemburg et, plus près de nous, Pigou, Robinson, Schumpeter...

10 À savoir, entre autres, les chapitres IX-1 (Hume), XVI-5 (Proudhon), XVII (Marx) et XXIII (Jevons et Marshall).

11 Citons, par exemple, les chapitres III-VI (les «mercantilistes»), XXI (Les origines de la pensée néoclassique), XXX (Veblen), XXXVII (La théorie de l'équilibre général depuis 1939).
} 
quelque sorte plus pédagogiques et qu'un dernier lot de contributions ${ }^{12}$, enfin, tente d'accorder un crédit de cohérence aux auteurs envisagés. Cette hétérogénéité dans le recul critique, à laquelle se joint une appréhension tout aussi variée de la littérature secondaire, peut être rapprochée des nuances méthodologiques ${ }^{13}$ qui président aux commentaires; lesquelles nuances sont du reste revendiquées par les éditeurs dans l' « Avant-propos » de l'ouvrage, parce qu'elles constitueraient une « richesse » (ibid., p. 12). Il ne faut cependant pas se faire d'illusions : une telle diversité méthodologique et surtout critique peut désorienter un étudiant de premier cycle, sauf si celui-ci a un intérêt vraiment très prononcé pour l'histoire de la pensée économique. À l'évidence, la Nouvelle Histoire... s'adresse avant tout au lecteur disposant d'un certain bagage en ce domaine. Ce dernier y trouvera une mine d'informations sans pareil. Certes, on a déjà souligné l'absence ou le peu d'évocation de certains courants, thèmes ou auteurs. Il est néanmoins particulièrement appréciable que de nombreux commentateurs signalent l'envergure limitée de leur propre champ d'étude ${ }^{14}$, tout en fournissant, pour la plupart, une riche bibliographie permettant au lecteur de compléter ses connaissances. On ne peut, en somme, être plus royaliste que le roi et, à ce jour, l'ouvrage dirigé par Béraud et Faccarello constitue la plus foisonnante et la plus stimulante des histoires de notre discipline.

\footnotetext{
${ }^{12}$ Nous pensons en particulier aux chapitres XII (Smith), XXIV (Walras) et XXXIV (Keynes).

${ }^{13}$ L'illustration la plus prononcée de ces nuances résulte de la comparaison que l'on peut faire entre les deux séries d'études suivantes : d'une part, les commentaires consacrés à Law, Malthus, Ricardo, Say, Smith (chapitres VII, XII, XIII), marqués pour tout ou partie par l'emploi d'une terminologie contemporaine, du style mathématique et par le souci de faire de ces auteurs des précurseurs d'économistes du $\mathrm{XX}^{\mathrm{e}}$ siècle; d'autre part, les contributions relatives aux «mercantilistes" (chapitres III-VI) et, dans un tout autre registre, aux controverses en théorie de l'entreprise et à la théorie des préférences révélées (chapitre XXXVI), respectivement caractérisées par de nombreuses « mises en contexte » et le refus de recourir à un mode d'exposition mathématique.

${ }^{14}$ Voir t. I, p. 16, 19-20, 71, 114, 145, 170, 226, 256, 281, 433, 559-561 ; t. II, p. 13, 75, 80, 148, 161, 171, 193, 203, 256, $273,499,525,557,573,590$ (n. 13) ; t. III, p. 65, 237-238, 336, 340.
} 


\section{BIBLIOGRAPHIE}

- Béraud A. et Faccarello G. (1992-2000), Nouvelle histoire de la pensée économique - t. I (1992) : Des scolastiques aux classiques; t. II (2000): Des premiers mouvements socialistes aux néoclassiques; t. III (2000): Des institutionnalistes à la période contemporaine, Paris, La Découverte.

- Blaug M. (1985), La pensée économique - Origine et développement, 4 e éd., Paris, Economica.

- Dupont de Nemours P. S. (1767), « De l'origine et des progrès d'une science nouvelle », in Daire E. éd., Physiocrates - Première partie (1846), Genève, Slatkine Reprints, 1971, p. 335-366.

- $\quad$ Ekelund R. B. et Hebert R. F. (1983), A History of Economic Theory and Method, Mc Graw Hill.

- Eltis W. (1993), « [Compte rendu de Béraud A. et Faccarello G. (1992)] », The European Journal of the History of Economic Thought, vol. 1, n¹, Automne, p. 161-166.

- Gide C. et Rist C. (1944), Histoire des doctrines économiques depuis les physiocrates jusqu'à nos jours, 6éd., Paris, Dalloz, 2000.

- Pribram K. (1983), Les fondements de la pensée économique, Paris, Economica, 1986.

- Schumpeter J. A. (1954), Histoire de l’analyse économique, Gallimard, 1983, 3 t..

- Smith A. (1763), «Early Draft of Part of the Wealth of Nations », Lectures on Jurisprudence, Oxford University Press, 1978, p. 562-581.

- Smith A. (1762-1763 et 1763-1764), Lectures on Jurisprudence, Oxford University Press, 1978.

- Smith A. (1776), Enquête sur la nature et les causes de la richesse des nations, trad. P. Taieb, Paris, Presses Universitaires de France, 1995, 4 vol.. 